EPJ Web of Conferences 116, 01001 (2016)

DOI: $10.1051 /$ epjconf/201611601001

(C) Owned by the authors, published by EDP Sciences, 2016

\title{
Multi-PMT Optical Module Designs for IceCube-Gen2
}

\author{
Alexander Kappes ${ }^{\mathrm{a}}$ \\ Erlangen Centre for Astroparticle Physics, Friedrich-Alexander-Universität Erlangen-Nürnberg, \\ 91058 Erlangen, Germany
}

\begin{abstract}
IceCube-Gen2 is the planned next generation neutrino telescope at the South Pole incorporating a high-energy array for neutrino astronomy and a dense array (PINGU) aimed at the determination of the neutrino mass hierarchy. Here, we present alternative designs to IceCube-Gen2's single-PMT baseline optical module which are currently being developed. These designs feature up to 24 smaller photomultipliers and use glass and gel with enhanced UV transparency to increase the number of detected photons and provide additional information. Thereby, they have the potential to significantly enhance the performance of IceCube-Gen2.
\end{abstract}

\section{Introduction}

The design of the Digital Optical Module (DOM) used in the IceCube detector [1] has proven to be highly reliable and, thereby, has played a key role in providing the high-quality data that enabled the recent discovery of high-energy cosmic neutrinos [2]. For the next-generation neutrino telescope in the deep ice at the South Pole, IceCube-Gen2, about 10,000 additional optical sensors will be deployed. These are required to operate reliably without maintenance over the full livetime of the detector of $10+$ years at depths down to $2,700 \mathrm{~m}$ with pressures up to $550 \mathrm{bar}^{1}$ and temperatures down to $-45 \mathrm{C}^{\circ}$. In view of these harsh environmental conditions, the baseline design for IceCube-Gen2 is an advancement of the successful IceCube-DOM design. While all parts exposed to the high pressure are retained, developments in recent years allow to significantly simplify the readout electronics, thereby reducing complexity and power consumption [3].

Like the original DOM, this new design features a single large 10" photomultiplier (PMT) pointing downwards, resulting in a very asymmetric angular acceptance. In particular, in view of the recent success in observing cosmic neutrinos from the Southern hemisphere [2], i.e. detecting events entering the detector from above, this strongly downward peaked sensitivity is an undesirable feature ${ }^{2}$. In addition, the single-PMT design doesn't provide any information on the arrival directions of the detected photons, and the absorption of photons by the current DOM glass increases rapidly as the wavelength of

\footnotetext{
${ }^{a}$ e-mail: kappes@icecube.wisc.edu

1 This pressure is reached during freeze-in.

${ }^{2}$ In fact, the IceCube DOM also has some sensitivity to photons produced above the optical module due to backscattering in the ice. However, scattered photons lose directional information on their origin.

This is an Open Access article distributed under the terms of the Creative Commons Attribution License 4.0, which permits unrestricted use, distribution, and reproduction in any medium, provided the original work is properly cited.
} 

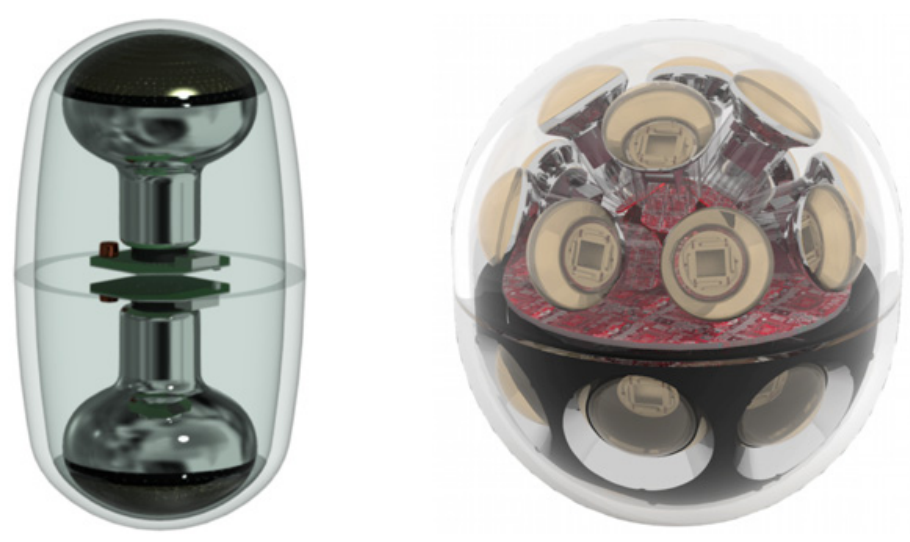

Figure 1. Renderings of the D-Egg (left, taken from [4]) and mDOM (right) concepts for IceCube-Gen2. In case of the latter, the top part of the black PMT holding structure visible in the lower half has been removed.

photons decreases below $400 \mathrm{~nm}$, thereby losing the UV photons from the Cherenkov spectrum which rises with $1 / \lambda^{2}$. Therefore, in parallel to the development of this baseline design, new detector concepts are being explored that aim at improving on these and other features. Two of them are the so-called D-Egg [4] and mDOM [5] concepts which use multiple PMTs and glass with improved UV transparency. Another concept, discussed in [6], uses large surfaces of wavelength shifters to significantly enhance the number of detected photons at low costs.

\section{The D-Egg and mDOM concepts}

The idea of optical sensors with multiple PMTs was first proposed in 1979 in the framework of the DUMAND project [7]. The D-Egg concept, depicted in Fig. 1 left, incorporates two oppositely oriented 8" PMTs (R5912-100 HQE) housed in an ellipsoidal pressure vessel. At its widest part, the pressure vessel measures $300 \mathrm{~mm}(\sim 12 ")$ and, hence, is about 1" narrower than the standard IceCube DOM. This allows for drilling narrower holes into the ice, thereby saving fuel costs and drilling time. First pressure tests up to 700 bar including penetrator, a dummy PMT and optical gel were successful. The observed bubble formation in the gel is under investigation. The glass of the pressure vessel as well as the optical gel for coupling the PMTs to the glass have been developed to provide increased UV transparency. For readout of each PMT, the analog waveforms will be continuously sampled with a fast ADC.

The mDOM concept (Fig. 1 right), which is based on the KM3NeT optical module [8], pushes the multi-PMT concept even further and incorporates 24 3" PMTS (HamamatsuR12199-02) into a pressure vessel of $358 \mathrm{~mm}$ (14") diameter. The large number of PMTs allows to gain information on the direction of a light source already on the single module level and coincidences between PMTs can, e.g, be used for suppression of background. Each PMT has a base attached to it that, in addition to containing electronics that generates the high voltage via a Cockcroft Walton circuit ${ }^{3}$, performs advanced signal processing. For readout of the 24 PMTs, a multi-level comparator design with up to 63 thresholds is envisioned which samples the signal of each PMT already on the PMT base. Each comparator will provide the leading-edge time and the time over threshold.

\footnotetext{
${ }^{3}$ Developed by Nikhef for the KM3NeT optical module.
} 


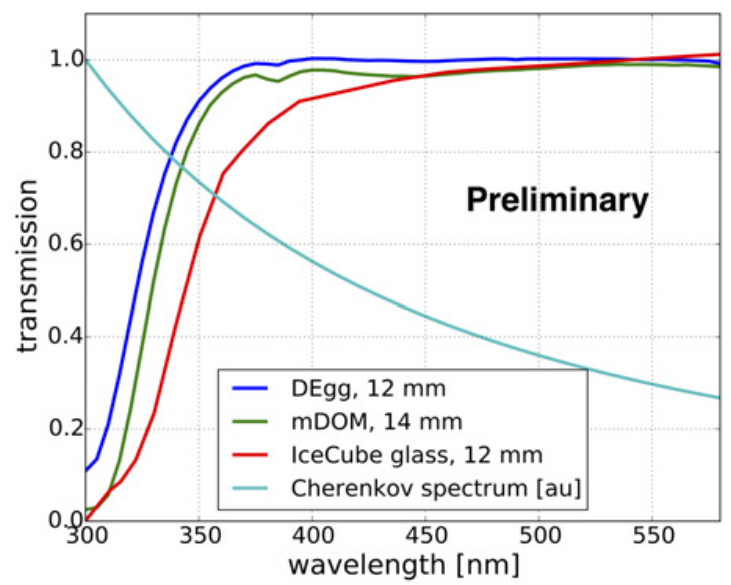

Figure 2. Transmissivity of different pressure vessel glasses as function of wavelength. The measurement for the D-Egg and mDOM glasses were performed in air and losses due to reflection deducted. For illustrative purposes, the $1 / \lambda^{2}$ Cherenkov spectrum is also shown with an arbitrary scale.

\subsection{UV sensitivity}

Due to the Cherenkov spectrum increasing with $1 / \lambda^{2}$, already minor extensions of the sensitivity to shorter wavelengths increases the number of detected photons significantly. The achievable gain is weakened by the fact that both scattering and absorption length rise below $\sim 400 \mathrm{~nm}$. However, a sizable gain remains even for larger distances between photon source and optical module.

For the IceCube DOM, the limiting factor here is the transmissivity of the pressure vessel which starts to significantly absorb photons already at $400 \mathrm{~nm}$ (see Fig. 2) and reaches $50 \%$ transmissivity around $345 \mathrm{~nm}$. The glass of the D-Egg, specifically developed to provide better UV transparency by reducing its $\mathrm{Fe}_{2} \mathrm{O}_{3}$ concentration, features a significantly lower cutoff wavelength and reaches 50\% around $320 \mathrm{~nm}$. In particular, it also maintains its maximum transmissivity to much lower wavelengths. The mDOM glass, which is the standard glass used by the manufacturer (NAUTILUS Marine Service $\mathrm{GmbH}$ ) for their deep-see pressure vessels, reaches $50 \%$ transmissivity around $330 \mathrm{~nm}$, thereby being only slightly worse than the special UV-transparent glass. Both glasses therefore represent attractive alternatives to the one used in the current optical modules. Their contamination with radioactive elements still needs to be determined carefully though, as this has a large impact on the dark count rate of the PMTs in the otherwise very radioactive pure environment in the deep ice.

\subsection{Effective areas}

In addition to an increased UV transparency, both new concepts also feature increased photocathode area (D-Egg: $\sim 2 \times 320 \mathrm{~cm}^{2}=640 \mathrm{~cm}^{2}$; $\mathrm{mDOM}: \sim 24 \times 50 \mathrm{~cm}^{2}=1200 \mathrm{~cm}^{2}$ ) compared to the current IceCube DOM with $\sim 500 \mathrm{~cm}^{2}$. Also, both the Gen2 baseline design and the D-Egg concept foresee the usage of super-bialkali photocathode material which provides up to $\sim 20 \%$ higher photon detection efficiency. Whether this also poses a cost-effective option for the 3" PMTs used in the mDOM has to be evaluated. Figure 3 shows the effective areas for the D-Egg and mDOM concepts including quantum efficiency. Compared to the single-PMT baseline design (not shown), the D-Egg shows a significantly reduced angular dependence with a maximum reduction of about $20 \%$. Owing to its large number of PMTs, the mDOM features an even more uniform angular acceptance with a maximum variation of 

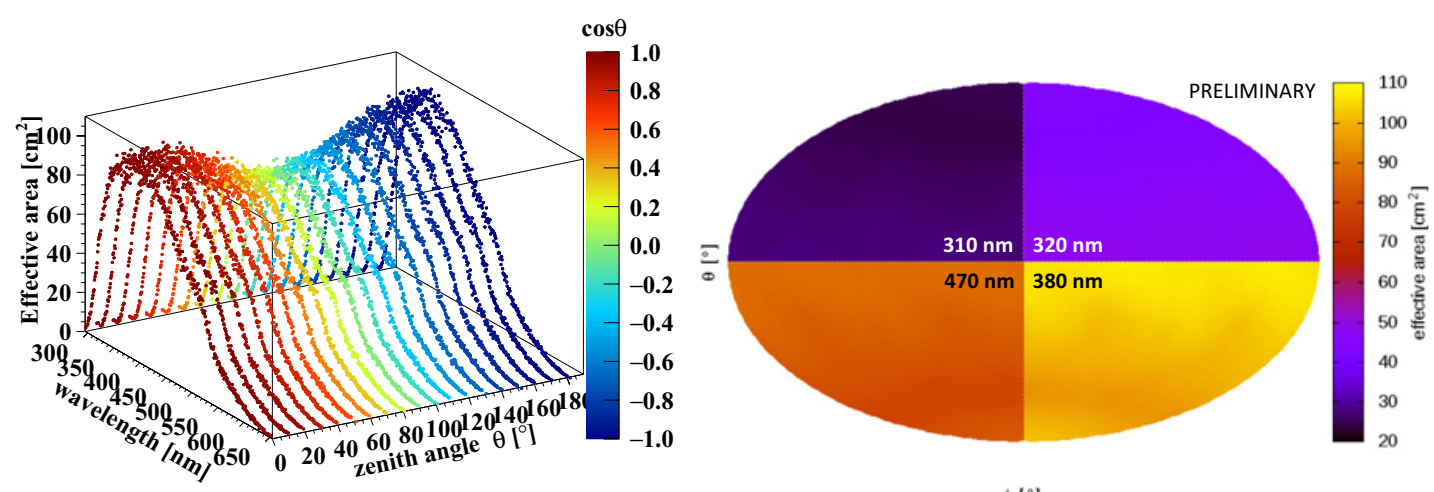

$\phi\left[^{\circ}\right]$

Figure 3. Left: Effective area of the D-Egg including PMT quantum efficiency as a function of wavelength for different photon zenith angles (module orientation as in Fig. 1 left). Taken from [4]. — Right: Hammer projection of the mDOM photon acceptance including PMT quantum efficiency represented as effective area as a function of direction of the incident photons in zenith and azimuth angle (module orientation as in Fig. 1 right). Values are shown for different photon wavelengths. Due to the symmetry in the orientation of the PMTs, each shown quadrants is representative of the total solid angle for the respective wavelength.

$12 \%$. Both new concepts therefore improve significantly on the amount of effective area per optical module and its uniformity in solid angle. Detailed measurements and simulations currently underway will allow to quantify the impact of these improvements on the detector performance.

\section{Conclusions}

Parallel to the advancement of the proven IceCube DOM design, several alternative designs for the next generation neutrino telescope at the South Pole, IceCube-Gen2, are being investigated. These designs improve on several aspects of the Gen-2 baseline design, in particular the uniformity of the angular acceptance and the overall photon acceptance. The aim is to develop prototypes within the next two years which can be tested under real conditions in the deep ice at the South Pole in advance of the installation of the actual IceCube-Gen2 detector. A first mechanical design for both sensor types has been completed and the development of specific read-out electronics has commenced. Conceptual prototypes are planned for 2016. Simulations currently undertaken address the impact of the utilization of the new module concepts on the performance of IceCube-Gen2 which is expected to benefit from the increased effective area and superior information on the recorded photons. In the end, a combination of different module types may turn out to be most effective.

\section{References}

[1] F. Halzen, S.R. Klein, Rev. Sci. Instrum. 81, 081101 (2010), arXiv: 1007.1247

[2] M. Aartsen et al. (IceCube), Phys. Rev. Lett. 113, 101101 (2014), arXiv: 1405.5303

[3] M.G. Aartsen et al. (IceCube-Gen2), Generation 2 IceCube Digital Optical Module and DAQ, in Proc. of the 34th International Cosmic Ray Conference (The Hague, The Netherlands, 2015), arXiv: 1510.05228

[4] M.G. Aartsen et al. (IceCube-Gen2), A dual-PMT optical module (D-Egg) for IceCube-Gen2, in Proc. of the 34th International Cosmic Ray Conference (The Hague, The Netherlands, 2015), arXiv: 1510.05228 
[5] M.G. Aartsen et al. (IceCube-Gen2), Multi-PMT optical modules for IceCube-Gen2, in Proc. of the 34th International Cosmic Ray Conference (The Hague, The Netherlands, 2015), arXiv: 1510.05228

[6] D. Hebecker, A wavelength-shifting optical module (WOM) for in-ice neutrino detectors, these proceedings

[7] V.V. Borog et al., Measuring module for registration of Cherenkov radiation in the water, in Proc. of the 16th International Cosmic Ray Conference (1979), Vol. 8, p. 380

[8] H. Löhner et al. (KM3NeT), Nucl. Inst. Meth. A718, 513 (2013) 